\title{
Three Dimensional Curvilinear Structure Detection Using Optimally Oriented Flux
}

\author{
Max W.K. Law and Albert C.S. Chung \\ Lo Kwee-Seong Medical Image Analysis Laboratory, \\ Department of Computer Science and Engineering, \\ The Hong Kong University of Science and Technology, Hong Kong \\ \{maxlawwk, achung\}@ese.ust.hk
}

\begin{abstract}
This paper proposes a novel curvilinear structure detector, called Optimally Oriented Flux (OOF). OOF finds an optimal axis on which image gradients are projected in order to compute the image gradient flux. The computation of OOF is localized at the boundaries of local spherical regions. It avoids considering closely located adjacent structures. The main advantage of OOF is its robustness against the disturbance induced by closely located adjacent objects. Moreover, the analytical formulation of OOF introduces no additional computation load as compared to the calculation of the Hessian matrix which is widely used for curvilinear structure detection. It is experimentally demonstrated that OOF delivers accurate and stable curvilinear structure detection responses under the interference of closely located adjacent structures as well as image noise.
\end{abstract}

\section{Introduction}

Analysis of curvilinear structures in volumetric images has a wide range of applications, for instance centerline extraction [13], detection and segmentation [7 15 [9], vascular image enhancement [12|811] or visualization [2]. In particular, low-level detectors which are sensitive to curvilinear structures are the foundations of the aforementioned applications. One classic low-level detector is the multiscale based image intensity second-order statistics. Lindeberg [10] conducted in depth research regarding the use of the Gaussian smoothing function with various scale factors for extracting multiscale second-order statistics. Koller et al. [7] exploited the image intensity secondorder statistics to form Hessian matrices for the analysis of curvilinear structures in three dimensional image volumes. Frangi et al. [6] introduced the vesselness measure based on eigenvalues extracted from the Hessian matrix in a multiscale fashion. Krissian et al. [9] studied the relation between the Hessian matrix and the image gradient computed in multiple scales for the detection of tubular structures. Manniesing et al. [11] made use of the multiscale Hessian matrix based features to devise a nonlinear scale space representation of curvilinear structures for vessel image enhancement.

Another recently developed low-level detector for the curvilinear structure analysis is the image gradient flux. It is a scalar measure which quantifies the amount of image gradient flowing in or out of a local spherical region. A large magnitude of the image gradient flux is an indication of the presence of a curvilinear structure disregarding the

D. Forsyth, P. Torr, and A. Zisserman (Eds.): ECCV 2008, Part IV, LNCS 5305, pp. 368 382, 2008.

(C) Springer-Verlag Berlin Heidelberg 2008 
structure direction. Bouix et al. proposed to compute the image gradient flux for extracting centerlines of curvilinear structures [3]. Siddqi et al. [15] showed promising vascular segmentation results by evolving an image gradient flux driven active surface model. But the major disadvantage of the image gradient flux is its regardless of directional information.

Grounded on the multiscale based Hessian matrix, Sato et al. [12] presented a thorough study on the properties of the eigenvalues extracted from the Hessian matrix in different scales, and their performance in curvilinear structure segmentation and visualization. The study showed that the eigenvalues extracted from the Hessian matrix can be regarded as the results of convolving the image with the second derivative of a Gaussian function. This function offers differential effects which compute the difference between the intensity inside an object and in the vicinity of the object. However, if the intensity around the objects is not homogeneous due to the presence of closely located adjacent structures, the differential effect given by the second derivatives of Gaussian is adversely affected.

In this paper, we propose a novel detector of curvilinear structures, called optimally oriented flux (OOF). Specifically, the oriented flux encodes directional information by projecting the image gradient along some axes, prior to measuring the amount of the projected gradient that flows in or out of a local spherical region. Meanwhile, OOF discovers the structure direction by finding an optimal projection axis which minimizes the oriented flux. OOF is evaluated for each voxel in the entire image. The evaluation of OOF is based on the projected image gradient at the boundary of a spherical region centered at a local voxel. When the local spherical region boundary touches the object boundary of a curvilinear structure, the image gradient at the curvilinear object boundary produces an OOF detection response. Depending on whether the voxels inside the local spherical region have stronger intensity, the sign of the OOF detection response varies. It can be utilized to distinguish between regions inside and outside curvilinear structures.

The major advantage of the proposed method is that the OOF based detection is localized at the boundary of the local spherical region. Distinct from the Hessian matrix, OOF does not consider the region in the vicinity of the structure where a nearby object is possibly present. As such, OOF detection result is robust against the disturbance introduced by closely located objects. With this advantage, utilizing OOF for curvilinear structure analysis is highly beneficial when closely located structures are present. Moreover, the computation of OOF does not introduce additional computation load compared to the Hessian matrix. Validated by a set of experiments, OOF is capable of providing more accurate and stable detection responses than the Hessian matrix, with the presence of closely located adjacent structures.

\section{Methodology}

\subsection{Optimally Oriented Flux (OOF)}

The notion of oriented flux along a particular direction refers to the amount of image gradient projected along that direction at the surface of an enclosed local region. The image gradient can flow either in or out of the enclosed local region. Without loss of 
generality, our elaboration focuses on the situation where the structures have stronger intensity than background regions. As such, optimally oriented flux (OOF) aims at finding an optimal projection direction that minimizes the inward oriented flux for the detection of curvilinear structure.

The outward oriented flux along a direction $\hat{\rho}$ is calculated by projecting the image gradient $\boldsymbol{v}(\cdot)$ along the direction of $\hat{\rho}$ prior to the computation of flux in a local spherical region $S_{r}$ with radius $r$. Based on the definition of flux [13], the computation of the outward oriented flux along the direction of $\hat{\rho}$ is,

$$
f(\boldsymbol{x} ; r, \hat{\rho})=\int_{\partial S_{r}}((\boldsymbol{v}(\boldsymbol{x}+\boldsymbol{h}) \cdot \hat{\rho}) \hat{\rho}) \cdot \hat{n} d A,
$$

where $d A$ is the infinitesimal area on $\partial S_{r}, \hat{n}$ is the outward unit normal of $\partial S_{r}$ at the position $\hat{h}$. As $\partial S_{r}$ is a sphere surface, $\boldsymbol{h}=r \hat{n}$, thus

$$
f(\boldsymbol{x} ; r, \hat{\rho})=\int_{\partial S_{r}}\left\{\sum_{k=1}^{3} \sum_{l=1}^{3}\left(v_{k}(\boldsymbol{x}+r \hat{n}) \rho_{k} \rho_{l} n_{l}\right)\right\} d A=\hat{\rho}^{T} \mathbf{Q}_{r, \boldsymbol{x}} \hat{\rho},
$$

where $\hat{\rho}=\left(\rho_{1}, \rho_{2}, \rho_{3}\right)^{T}, \boldsymbol{v}(\boldsymbol{x})=\left(v_{1}(\boldsymbol{x}), v_{2}(\boldsymbol{x}), v_{3}(\boldsymbol{x})\right)^{T}, \hat{n}=\left(n_{1}, n_{2}, n_{3}\right)^{T}, \mathbf{Q}_{r, \boldsymbol{x}}$ is a matrix that the entry at the $i$ th row and $j$ th column $(i, j \in\{1,2,3\})$ is,

$$
q_{r, \boldsymbol{x}}^{i, j}=\int_{\partial S_{r}} v_{i}(\boldsymbol{x}+r \hat{n}) n_{j} d A .
$$

\subsection{Analytical Computation of OOF}

The idea of OOF is to identify the direction $\hat{p}$ that the inward oriented flux attains the minimum. It is not easy to discretize any one of the surface integrals of Equations 1 and 3 to estimate oriented flux and find the optimal axis which minimizes the inward oriented flux. Nevertheless, computation of OOF can be achieved analytically by acquiring the values of the entries of $\mathbf{Q}_{r, \boldsymbol{x}}$, that only involves convolving an image with a set of filters $\psi_{r, i, j}$,

$$
q_{r, \boldsymbol{x}}^{i, j}=\psi_{r, i, j}(\boldsymbol{x}) * I(\boldsymbol{x}) .
$$

The above formulation avoids discretization and reduces computation complexity as compared with the discretization of either Equation[1 or Equation 3 By using fast Fourier transform, the complexity of evaluating Equation 4 and thus $\mathbf{Q}_{r, x}$ is $O(N \log N)$, where $\forall \boldsymbol{x} \in \Omega$ and $\Omega$ is the image domain having $N$ voxels. The proposed method introduces no additional computation load compared to some traditional approaches, such as Hessian matrix based methods [12[9]6].

We begin the elaboration of the filters $\psi_{r, i, j}(\boldsymbol{x})$ from Equation 3 ,

$$
q_{r, \boldsymbol{x}}^{i, j}=\int_{\partial S_{r}} v_{i}(\boldsymbol{x}+r \hat{n}) n_{j} d A=\int_{\partial S_{r}}\left[v_{i}(\boldsymbol{x}+r \hat{n}) \hat{a_{j}}\right] \cdot \hat{n} d A,
$$

where $\hat{a_{1}}, \hat{a_{2}}$ and $\hat{a_{3}}$ are the unit vectors along the $\mathrm{x}-, \mathrm{y}$ - and z-directions respectively. Assuming that $v$ is continuous, by the divergence theorem,

$$
q_{r, \boldsymbol{x}}^{i, j}=\int_{S_{r}} \nabla \cdot\left[v_{i}(\boldsymbol{x}+\boldsymbol{y}) \hat{a}_{j}\right] d V=\int_{S_{r}} \frac{\partial}{\partial \hat{a}_{j}} v_{i}(\boldsymbol{x}+\boldsymbol{y}) d V,
$$


where $\boldsymbol{y}$ is the position vector inside the sphere $S_{r}$ and $d V$ is the infinitesimal volume in $S_{r}$. The continuous image gradient $\boldsymbol{v}(\boldsymbol{x})$ is acquired by convolving the discrete image with the first derivatives of Gaussian with a small scale factor, i.e. $v_{i}(\boldsymbol{x})=\left(g_{\hat{a_{i}}, \sigma} *\right.$ $I)(\boldsymbol{x})$, where $*$ is the convolution operator, $g_{\hat{a_{i}}, \sigma}$ is the first derivative of Gaussian along the direction of $\hat{a}_{i}$ and $\sigma=1$ in all our implementations. Furthermore, the volume integral of Equation 6 is extended to the entire image domain $\Omega$ by employing a step function, $b_{r}(\boldsymbol{x})=\left\{\begin{array}{l}1,\|\boldsymbol{x}\| \leq r, \\ 0, \text { otherwise }\end{array}\right.$, hence

$$
q_{r, \boldsymbol{x}}^{i, j}=\int_{\Omega} b_{r}(\boldsymbol{y})\left(\left(g_{\hat{a_{i}} \hat{a_{j}}, \sigma} * I\right)(\boldsymbol{x}+\boldsymbol{y})\right) d V=\left(\left(b_{r} * g_{\hat{a_{i}} \hat{a_{j}}, \sigma}\right)(\boldsymbol{x})\right) * I(\boldsymbol{x}),
$$

where $g_{\hat{a_{i}} \hat{a_{j}}, \sigma}$ is the second derivative of Gaussian along the axes $\hat{a_{i}}$ and $\hat{a_{j}}$. Therefore, the set of linear filters of Equation 4 is $\psi_{r, i, j}(\boldsymbol{x})=\left(b_{r} * g_{\hat{a_{i}}, \hat{a_{j}}, \sigma}\right)(\boldsymbol{x})$. The next step is to obtain the analytical Fourier expression of $\psi_{r, i, j}(\boldsymbol{x})$ in order to compute the convolution in Equation 4 by Fourier coefficient multiplication. Denote $\Psi_{r, i, j}(\boldsymbol{u})$ be the Fourier expression of $\psi_{r, i, j}(\boldsymbol{x})$, where $\boldsymbol{u}=\left(u_{1}, u_{2}, u_{3}\right)^{T}$ is the position vector in the frequency domain. The values of $u_{1}, u_{2}$ and $u_{3}$ are in "cycle per unit voxel" and in a range of $[-0.5,0.5)$. By employing Fourier transforms on $g_{\hat{a_{i}} \hat{a_{j}}, \sigma}$ and Hankel transforms [4] on $b_{r}(\boldsymbol{x})$,

$$
\Psi_{r, i, j}(\boldsymbol{u})=4 \pi r u_{i} u_{j} e^{-2(\pi\|\boldsymbol{u}\| \sigma)^{2}} \frac{1}{\|\boldsymbol{u}\|^{2}}\left(\cos (2 \pi r\|\boldsymbol{u}\|)-\frac{\sin (2 \pi r\|\boldsymbol{u}\|)}{2 \pi r\|\boldsymbol{u}\|}\right) .
$$

Based on the above formulation, the optimal projection axis which minimizes inward oriented flux can be computed analytically. Denote the optimal direction is $\boldsymbol{\omega}_{r, \boldsymbol{x}}$, minimizing inward oriented flux is equivalent to maximizing $f_{r}\left(\boldsymbol{x} ; \boldsymbol{\omega}_{r, \boldsymbol{x}}\right)$ subject to the constraint $\left\|\boldsymbol{\omega}_{r, \boldsymbol{x}}\right\|=\left[\boldsymbol{\omega}_{r, \boldsymbol{x}}\right]^{T} \boldsymbol{\omega}_{r, \boldsymbol{x}}=1$. The solution is found by taking the first derivative on the Lagrange equation,

$$
\mathcal{L}\left(\boldsymbol{\omega}_{r, \boldsymbol{x}}\right)=\left[\boldsymbol{\omega}_{r, \boldsymbol{x}}\right]^{T} \mathbf{Q}_{r, \boldsymbol{x}} \boldsymbol{\omega}_{r, \boldsymbol{x}}+\lambda_{r, \boldsymbol{x}}\left(1-\left[\boldsymbol{\omega}_{r, \boldsymbol{x}}\right]^{T} \boldsymbol{\omega}_{r, \boldsymbol{x}}\right),
$$

for $\nabla \mathcal{L}\left(\boldsymbol{\omega}_{r, \boldsymbol{x}}\right)=0$, and since $q_{r, \boldsymbol{x}}^{i, j}=q_{r, \boldsymbol{x}}^{j, i}$ (see Equation $\mathbf{7}$, and thus $\mathbf{Q}=\mathbf{Q}^{T}$,

$$
\mathbf{Q}_{r, \boldsymbol{x}} \boldsymbol{\omega}_{r, \boldsymbol{x}}=\lambda_{r, \boldsymbol{x}} \boldsymbol{\omega}_{r, \boldsymbol{x}}
$$

Equation 10 is in turn solved as a generalized eigenvalue problem. For volumetric images, there are at most three distinct pairs of $\lambda_{r, \boldsymbol{x}}$ and $\boldsymbol{w}_{r, \boldsymbol{x}}$. The eigenvalues can be positive, zero or negative. These eigenvalues are denoted as $\lambda_{i}(\boldsymbol{x} ; r)$, for $\lambda_{1}(\cdot) \leq \lambda_{2}(\cdot) \leq$ $\lambda_{3}(\cdot)$, and the corresponding eigenvectors are $\boldsymbol{\omega}_{i}(\boldsymbol{x} ; r)$. Inside a curvilinear structure having stronger intensity than the background, the first two eigenvalues would be much smaller than the third one, $\lambda_{1}(\cdot) \leq \lambda_{2}(\cdot)<<\lambda_{3}(\cdot)$ and $\lambda_{3}(\cdot) \approx 0$. The first two eigenvectors span the normal plan of the structure and the third eigenvector is the structure direction.

\subsection{Eigenvalues and Eigenvectors}

The major difference between the eigenvalues and eigenvectors extracted from $\mathrm{OOF}$ and those from the Hessian matrix is that the computation of OOF and thus, its eigenvalues and eigenvectors are grounded on the analysis of image gradient on the local 
sphere surface ( $\partial S_{r}$ in Equation 3). In contrast, as pointed out by Sato et al. [12], the computation of the Hessian matrix is closely related to the results of applying the second derivative of Gaussian function on the image. This function computes the weighted intensity average difference between the regions inside the structure and in the vicinity of the structure. As such, the coverage of this function extends beyond the boundary of target structures and possibly includes structures nearby. As a result, the weighted intensity average difference computed by the second derivative of Gaussian function can be affected by the adjacent objects. It can be harmful to the detection accuracy of the Hessian matrix when closely located adjacent structures are present.

On the contrary, the evaluation of OOF is performed on the boundary of a local spherical region $\partial S_{r}$. Detection response of OOF is induced from the intensity discontinuities at the object boundary when the local sphere surface touches the object boundary of the structure. The detection of OOF is localized at the boundary of the local spherical region. The localized detection avoids the inclusion of objects nearby. Therefore, the OOF based detection is robust against the disturbance introduced by closely located adjacent structures.

The eigenvalues extracted from OOF are the values of oriented flux along the corresponding eigenvectors,

$$
\lambda_{i}(\boldsymbol{x} ; r)=\left[\boldsymbol{\omega}_{i}(\boldsymbol{x} ; r)\right]^{T} \mathbf{Q}_{r, \boldsymbol{x}} \boldsymbol{\omega}_{i}(\boldsymbol{x} ; r)=f\left(\boldsymbol{x} ; r, \boldsymbol{\omega}_{i}(\boldsymbol{x} ; r)\right) .
$$

The image gradient at the object boundary of a strong intensity curvilinear structure points to the centerline of the structure. Inside the structure, when the local spherical region boundary $\partial S_{r}$ (see Equation 1) touches the object boundary, at the contacting position of these two boundaries, the image gradient $\boldsymbol{v}(\cdot)$ is aligned in the opposite direction of the outward normal $\hat{n}$, hence $\lambda_{1}(\cdot) \leq \lambda_{2}(\cdot)<<0$. On the other hand, the image gradient is perpendicular to the structure direction, the projected image gradient along $\boldsymbol{\omega}_{3}(\cdot)$ has zero or very small magnitude, thus $\lambda_{3}(\cdot) \approx 0$. In contrast, if OOF is computed for a voxel which is just outside the curvilinear structure, at the position where $\partial S_{r}$ touches the curvilinear structure boundary, the image gradient $\boldsymbol{v}(\cdot)$ is in the same direction as the outward normal $\hat{n}$. It results in a large positive eigenvalue, that is $\lambda_{3}(\cdot)>>0$.

Combining multiple eigenvalues to tailor a measure for identifying structures in a specific shape is now possible. For instance $\Lambda_{12}(\boldsymbol{x} ; r)=\lambda_{1}(\boldsymbol{x} ; r)+\lambda_{2}(\boldsymbol{x} ; r)$ can provide responses at the curvilinear object centerline with circular cross section. According to Equations 1 and 11 .

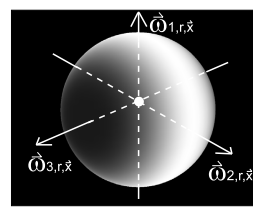

(a)

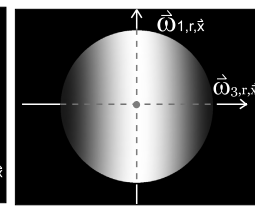

(b)

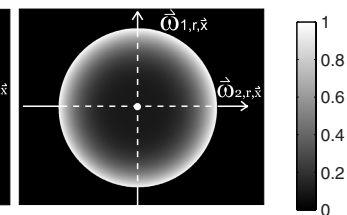

(c) (d)

Fig. 1. (a, b, c) The values of $\left\|\left[\mathbf{W}_{12}(\cdot)\right]^{T} \hat{n}\right\|$. (d) The intensity scale of the images in (a-c). 


$$
\Lambda_{12}(\boldsymbol{x} ; r)=\int_{\partial S_{r}}\left(\left[\mathbf{W}_{12}(\boldsymbol{x} ; r)\right]^{T} \boldsymbol{v}(\boldsymbol{x}+\boldsymbol{h})\right) \cdot\left(\left[\mathbf{W}_{12}(\boldsymbol{x} ; r)\right]^{T} \hat{n}\right) d A .
$$

where $\mathbf{W}_{12}(\boldsymbol{x} ; r)=\left[\boldsymbol{\omega}_{1}(\boldsymbol{x} ; r) \quad \boldsymbol{\omega}_{2}(\boldsymbol{x} ; r)\right]$. The term involving the projection of $\hat{n}$ in the second half of the surface integral of the above equation is independent to the image gradient. This term varies along the boundary of the spherical region $\partial S_{r}$. It is a weighting function that makes the projected image gradients at various positions on the sphere surface contribute differently to the resultant values of $\Lambda_{12}(\boldsymbol{x} ; r)$. The values of $\left\|\left[\mathbf{W}_{12}(\boldsymbol{x} ; r)\right]^{T} \hat{n}\right\|$ on the local spherical region surface are shown in Figures $1 \mathrm{~A}-\mathrm{c} . \mathrm{A}$ large value of $\left\|\left[\mathbf{W}_{12}(\boldsymbol{x} ; r)\right]^{T} \hat{n}\right\|$ represents the region where $\Lambda_{12}(\boldsymbol{x} ; r)$ is sensitive, as the projected image gradient at that region receives a higher weight for the computation of $\Lambda_{12}(\boldsymbol{x} ; r)$. The large valued regions of $\left\|\left[\mathbf{W}_{12}(\boldsymbol{x} ; r)\right]^{T} \hat{n}\right\|$ are distributed in a ring shape around the axis $\boldsymbol{\omega}_{3}(\boldsymbol{x} ; r)$. In a curvilinear structure having circular cross section, the image gradient at the object boundary points to the centerline of the structure. Therefore, at the centerline of the structure, $\Lambda_{12}(\boldsymbol{x} ; r)$ delivers the strongest response if $r$ and the radius of the structure are matched.

Finally, it is worth mentioning that the elaboration of $\Lambda_{12}(\cdot)$ merely demonstrates a possibility to integrate different eigenvalues to facilitate the analysis of curvilinear structures. It is possible to devise other combinations of eigenvalues of the proposed method analogous to those presented in [12] and [6].

\subsection{Regarding Multiscale Detection}

Multiscale detection is an essential technique for handling structures with various sizes. The multiscale detection of OOF involves repetitive computations of OOF using a set of radii ( $r$ in Equation 1). The radius set should cover both the narrowest and the widest curvilinear structures in an image volume. Since the evaluation of OOF is localized at the spherical region boundary, the spherical region has to touch the target structure boundary to obtain detection responses of OOF. As such, linear radius samples should be taken for OOF with the consideration of the voxel length in order to properly detect vessels in a given range of radii. It also ensures that a structure with non-circular cross section can induce detection responses of OOF obtained in at least one radius sample. We suggest that radius samples are taken in every 0.5 voxel length according to the Nyquist sampling rate.

For different values of $r$, the area covered by the surface integral of Equation 1 varies. Dividing the computation result of Equation 1 by $4 \pi r^{2}$ (the surface area of the spherical region) is an appropriate mean to normalize the detection response over radii and hence, the computation of Equation 1 is scale-invariant. Such normalization is essential to aggregating OOF responses in a multiple scale setting. For the same reason, the eigenvalues of $\mathbf{Q}_{r, \boldsymbol{x}}, \lambda_{i}(r, \boldsymbol{x})$ are divided by $4 \pi r^{2}$ prior to being utilized in any multiscale framework. This OOF normalization scheme is distinct to the averageoutward-flux (AOF) measure [5], which divides the outward flux by the surface area of the spherical region to attain the AOF-limiting-behavior. The AOF measure works only on the gradient of a distance function of a shape with its boundary clearly delineated. $\mathrm{OOF}$, in contrast, is applied to a gradient of a gray-scale image, where no explicit shape boundary is embedded and noise is possibly present. 


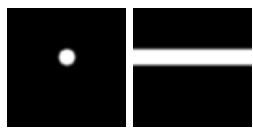

(a)

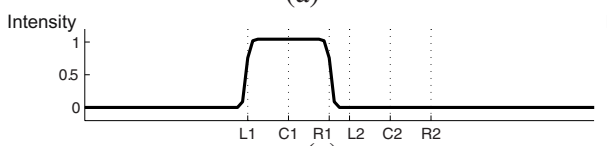

(c)

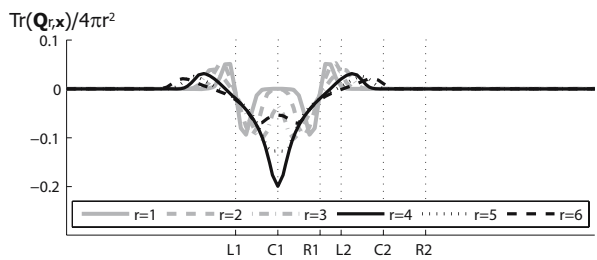

(e)

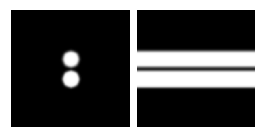

(b)

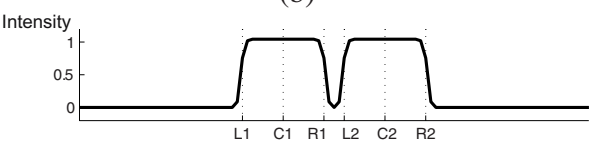

(d)

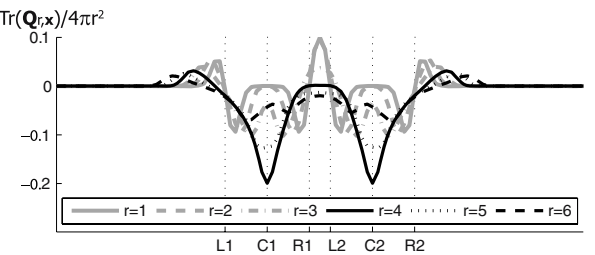

(f)

Fig. 2. Examples of evaluating OOF using multiple radii. (a, b) The slices of $z=0$ (left) and $x=0$ (right) of two synthetic image volumes consisting of synthetic tubes with a radius of 4 voxel length. $\mathrm{C} 1$ and $\mathrm{C} 2$ are the positions of the centers of the tubes. L1, R1 and L2, R2 are the positions of the boundaries of the tubes centered at $\mathrm{C} 1$ and $\mathrm{C} 2$ respectively. (b) The width of the separation between the closely located tubes is 2 voxel length. (c, d) The intensity profiles along the line $x=0, z=0$ of the synthetic image volumes shown in (a) and (b) respectively. (e, $\mathrm{f}$ ) The normalized trace of $\mathbf{Q}_{r, \boldsymbol{x}}$ along the line $x=0, z=0$ of the image volumes shown in (a) and (b) respectively.

In Figures $2 \mathrm{a}-\mathrm{f}$, we show two examples of evaluating OOF on image volumes consisting of one synthetic tube (Figures $2 \mathrm{a}$ and c) and two closely located synthetic tubes (Figures 2b and d) using multiple radii. The normalized trace of the matrix $\mathbf{Q}_{r, \boldsymbol{x}}$ (Equations 9], which is equal to the sum of the normalized eigenvalues of $\mathbf{Q}_{r, \boldsymbol{x}}$, is utilized to quantify the detection response strength of OOF. The normalized trace of the matrix $\mathbf{Q}_{r, \boldsymbol{x}}$ is computed using multiple radii in both of the synthetic image volumes. In Figures $2 \mathrm{e}$ and $\mathrm{f}$, it is observed that the normalized trace of $\mathbf{Q}_{r, \boldsymbol{x}}$ is negative for all radii inside the tubes. It attains its maximal negative values at the tube centers and with the radius $r$ matching the tube radius, i.e. $r=4$. The magnitudes of the normalized trace of $\mathbf{Q}_{r, \boldsymbol{x}}$ with $r=4$ decline at positions away from the tube centers. In these positions, it attains its maximal magnitudes with smaller values of $r$ when approaching the tube boundaries. Therefore, making use of the normalized trace of $\mathbf{Q}_{r, \boldsymbol{x}}$ as well as the normalized eigenvalues of $\mathbf{Q}_{r, \boldsymbol{x}}$, (the trace of $\mathbf{Q}_{r, \boldsymbol{x}}$ is equal to the sum of its eigenvalues), with maximal negative values or maximal magnitudes over radii is capable of delivering a strong detection responses inside curvilinear structures.

When OOF is computed using multiple radii, the spherical regions of OOF with large radii possibly overshoot the narrow structure boundaries. The computation of OOF with overshot radii can include the objects nearby and adversely affects the detection responses of OOF (see Figure 2e, $r=5$ and 6 versus Figure $2 \mathrm{f}, r=5$ and 6 ). In which, utilizing the normalized eigenvalues or the normalized trace of the matrix $\mathbf{Q}_{r, \boldsymbol{x}}$ with the maximal negative values or maximal magnitudes over radii as mentioned above can 
eliminate the responses obtained by using overshot radii. Furthermore, it excludes the OOF responses associated with undersized radii at the center of curvilinear structures (see Figures 2e and f, $r=1,2$ and 3 ). In the case that the radius of the spherical region $r$ matches the target structures, OOF avoids the inclusion of objects nearby. It therefore reports the same response at the centerlines of the tubes with $r=4$ despite the presence of closely located structures (see Figure 2k, $r=4$ versus Figure 2 ; $r=4$ ).

\section{Experimental Results}

In this section, we compare the performance of OOF and the Hessian matrix by using both synthetic data and real clinical cases. The differential terms of the Hessian matrix are obtained by employing the central mean difference scheme on the image smoothed by a Gaussian kernel with scale factor $\varrho$. The eigenvalues and eigenvectors extracted from the Hessian matrix and $\mathbf{Q}$ for OOF (Equation 10) are represented as $\lambda_{i}^{\mathbf{H}}(\boldsymbol{x} ; r)$, $\boldsymbol{\omega}_{i}^{\mathbf{H}}(\boldsymbol{x} ; r)$ and $\lambda_{i}^{\mathbf{Q}}(\boldsymbol{x} ; r), \boldsymbol{\omega}_{i}^{\mathbf{Q}}(\boldsymbol{x} ; r)$, respectively. The order of the eigenvalues and the notation of sums of the first two eigenvalues $\left(\Lambda_{12}^{\mathbf{H}}(\boldsymbol{x} ; r)\right.$ and $\left.\Lambda_{12}^{\mathrm{Q}}(\boldsymbol{x} ; r)\right)$ are analogous to those described in Section 2.2 .

\subsection{Synthetic Data}

The proposed method, OOF, is examined in this section using synthetic images containing tori with various sizes. There are 10 synthetic volumetric images in the size of $100 \times 100 \times 100$ voxels being generated for the synthetic experiments. The main purpose is to verify the performance of OOF and compare OOF with the Hessian matrix when closely located structures are present.

The configurations of the tori in the synthetic images are shown in Figure 3 . The number of tori in different synthetic images varies and depends on the values of $d$ and $R$. The tori are placed in a layer fashion along the $\mathrm{z}$-direction. The strategy to generate the first layer of tori is to place a torus with $D=10$ at the altitude $z=8$. The center

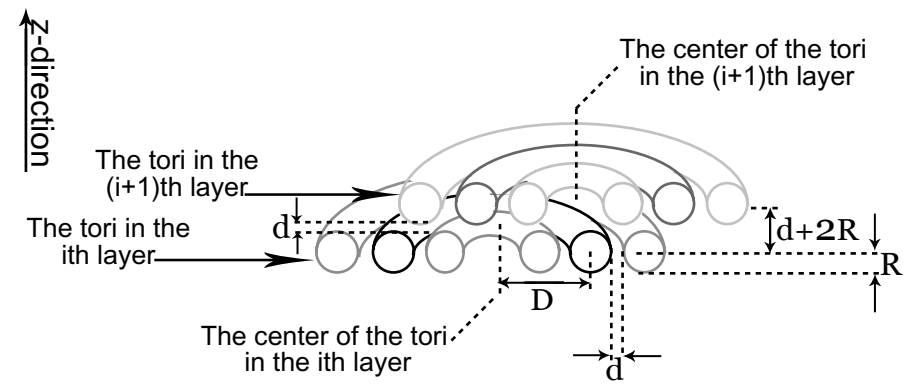

Fig. 3. The description of the tori. These tori have been used in the synthetic data experiments. The center of the tori in each layer is randomly selected from the positions of $(x=35, y=$ $35),(x=45, y=35),(x=35, y=45)$ and $(x=45, y=45)$. The values of $d$ and $R$ are fixed to generate a torus image. In the experiments, there are 10 torus images generated by using 10 pairs of $\{d, R\},\{2,1\},\{2,2\},\{2,3\},\{2,4\},\{2,5\},\{5,1\},\{5,2\},\{5,3\},\{5,4\}$ and $\{5,5\}$. 
of that torus is randomly selected among the positions $(x=45, y=45, z=8)$, $(x=35, y=45, z=8),(x=45, y=35, z=8)$ and $(x=35, y=35, z=8)$. We keep deploying adjacent tori centered at the same position of the first torus but having larger values of $D$ in an interval of $2 R+d$ until $D \leq 42$. Each successive layer of tori is generated in a $2 R+d$ interval of altitude $z$ for $z \leq 90$. The center of each layer of tori is randomly selected among the positions of $(x=35, y=35),(x=45, y=35)$, $(x=35, y=45)$ and $(x=45, y=45)$. The background intensity of these images is 0 and the intensity inside the tori is assigned to 1 . The torus images are smoothed by a Gaussian kernel with scale factor 1 to mimic the smooth intensity transition from structures to background. Each synthetic image is corrupted by two levels of additive Gaussian noise, with standard deviations of $\sigma_{\text {noise }}=\{0.75,1\}$. Finally, 20 testing cases are generated for this experiment.

The experiment results are based on the measures obtained in the estimated object scales of the both methods. For the testing objects with circular cross section such as the tori used in this experiment, computing the sums of the first two eigenvalues $\Lambda_{12}^{\mathbf{H}}(\cdot)$ and $\Lambda_{12}^{\mathrm{Q}}(\cdot)$ at structure centerlines is useful to determine the structure scales. The reason is that $\Lambda_{12}^{\mathrm{H}}(\cdot)$ of the Hessian matrix quantifies the second order intensity change occurred along the radial direction of a circle on the normal plane of the structure. Meanwhile, for OOF, $\Lambda_{12}^{\mathrm{Q}}(\cdot)$ evaluates the amount of gradient pointing to the centerlines of tubes with circular cross section. Based on the above observation, the object scale is obtained as $S_{\boldsymbol{x}}^{\mathbf{H}}=\arg \max _{s \in E}\left(-\frac{s^{2}}{3} \Lambda_{12}^{\mathbf{H}}\left(\boldsymbol{x} ; \frac{s}{\sqrt{3}}\right)\right)$ for the Hessian matrix (see [7]14] for details regarding the structure scale detection and [10] for Hessian matrix based feature normalization over scales) and $S_{\boldsymbol{x}}^{\mathbf{Q}}=\arg \max _{s \in F}\left(-\frac{1}{4 \pi s^{2}} \Lambda_{12}^{\mathbf{Q}}(\boldsymbol{x} ; s)\right)$ for OOF. The set of discrete detection scales of OOF and detection scales of the Hessian matrix are represented as $F$ and $E$ respectively. These scales cover the structure radii ranged from 1 to 6 voxel length. The radii of OOF are taken for each 0.5 voxel length and there are in total 11 different radii in $F$. Meanwhile, the same number of scales are logarithmically sampled for the Hessian matrix scale set $E$ so as to minimize the detection error of the Hessian matrix [12].

There are two measures being studied for the comparison of OOF and the Hessian matrix, "Angular discrepancy" and "Response fluctuation". For objects with circular cross section and having stronger intensity than the background, the third eigenvector represents the structure direction. At the estimated structure scales, we measure the angular discrepancy of the Hessian matrix and OOF by

$$
\arccos \left(\left|\boldsymbol{G}_{\boldsymbol{t}} \cdot \boldsymbol{\omega}_{3}^{\mathbf{H}}\left(\boldsymbol{x} ; S_{\boldsymbol{t}}^{\mathbf{H}}\right)\right|\right), \arccos \left(\left|\boldsymbol{G}_{\boldsymbol{t}} \cdot \boldsymbol{\omega}_{3}^{\mathbf{Q}}\left(\boldsymbol{x} ; S_{\boldsymbol{t}}^{\mathbf{Q}}\right)\right|\right),
$$

respectively, where $G_{t}$ is the ground truth direction, which is defined as the tangent direction of the torus inner-tube centerline at the position $t, t \in \mathcal{T}$, where $\mathcal{T}$ is a set of samples taken in every unit voxel length at the inter-tube centerlines of the tori. Bilinear interpolation is applied if $\boldsymbol{t}$ does not fall on an integer coordinate. The value of the angular discrepancy is in a range of $[0, \pi / 2]$ and a small value of the angular discrepancy represents an accurate estimation of structure direction.

The second measure, "Response fluctuation" for the tori having circular cross section is defined as the ratio between the variance and the mean absolute value of $\Lambda_{12}(\cdot)$. The "Response fluctuation" of the Hessian matrix and OOF are defined as 
Table 1. The performance of optimally oriented flux and the Hessian matrix obtained in the synthetic data experiments. The entries in the columns of "Angular discrepancy" include two values, the mean and the standard deviation (the bracketed values) of the resultant values of Equation 12 The values in the columns of "Response fluctuation" are the results based on Equation 13 .

\begin{tabular}{|l||c|c||c|c|}
\hline \multicolumn{5}{|c|}{$d=5, \sigma_{\text {noise }}=0.75$} \\
\hline & \multicolumn{2}{|c|}{ Angular discrepancy } & Response fluctuation \\
\hline R & Hessian matrix & OOF & Hessian matrix & OOF \\
\hline 1 & $0.406(0.250)$ & $0.309(0.176)$ & 0.270 & 0.246 \\
2 & $0.232(0.197)$ & $0.180(0.093)$ & 0.166 & 0.160 \\
3 & $0.109(0.111)$ & $0.110(0.065)$ & 0.092 & 0.095 \\
4 & $0.063(0.068)$ & $0.062(0.054)$ & 0.059 & 0.054 \\
5 & $0.054(0.075)$ & $0.059(0.027)$ & 0.052 & 0.056 \\
\hline
\end{tabular}

\begin{tabular}{|l||c|c||c|c|}
\hline \multicolumn{5}{|c|}{$d=2, \sigma_{\text {noise }}=0.75$} \\
\hline & \multicolumn{2}{|c|}{ Angular discrepancy } & Response fluctuation \\
\hline R & Hessian matrix & OOF & Hessian matrix & OOF \\
\hline 1 & $0.408(0.260)$ & $0.304(0.178)$ & 0.283 & 0.252 \\
2 & $0.305(0.215)$ & $0.227(0.129)$ & 0.218 & 0.195 \\
3 & $0.162(0.155)$ & $0.135(0.072)$ & 0.133 & 0.117 \\
4 & $0.098(0.127)$ & $0.087(0.055)$ & 0.092 & 0.085 \\
5 & $0.079(0.125)$ & $0.065(0.033)$ & 0.086 & 0.069 \\
\hline
\end{tabular}

\begin{tabular}{|l||c|c||c|c|}
\hline \multicolumn{5}{|c|}{$d=5, \sigma_{\text {noise }}=1$} \\
\hline & \multicolumn{2}{|c|}{ Angular discrepancy } & \multicolumn{2}{|c|}{ Response fluctuation } \\
\hline $\mathrm{R}$ & Hessian matrix & OOF & Hessian matrix & OOF \\
\hline 1 & $0.518(0.288)$ & $0.409(0.239)$ & 0.321 & 0.291 \\
2 & $0.331(0.252)$ & $0.246(0.148)$ & 0.210 & 0.200 \\
3 & $0.204(0.218)$ & $0.169(0.109)$ & 0.129 & 0.105 \\
4 & $0.112(0.158)$ & $0.110(0.080)$ & 0.089 & 0.080 \\
5 & $0.107(0.159)$ & $0.082(0.044)$ & 0.073 & 0.061 \\
\hline
\end{tabular}

\begin{tabular}{|l||c|c||c|c|}
\hline \multicolumn{5}{|c|}{$d=2, \sigma_{\text {noise }}=1$} \\
\hline & \multicolumn{2}{|c|}{ Angular discrepancy } & Response fluctuation \\
\hline $\mathrm{R}$ & Hessian matrix & OOF & Hessian matrix & $\mathbf{O O F}$ \\
\hline 1 & $0.532(0.305)$ & $0.414(0.243)$ & 0.338 & 0.298 \\
2 & $0.435(0.278)$ & $0.319(0.192)$ & 0.272 & 0.239 \\
3 & $0.279(0.243)$ & $0.200(0.132)$ & 0.177 & 0.134 \\
4 & $0.181(0.220)$ & $0.125(0.095)$ & 0.127 & 0.108 \\
5 & $0.157(0.217)$ & $0.097(0.088)$ & 0.107 & 0.085 \\
\hline
\end{tabular}

$$
\frac{\operatorname{Var}_{\boldsymbol{t} \in \mathcal{T}}\left(\Lambda_{12}^{\mathbf{H}}\left(\boldsymbol{x} ; S_{\boldsymbol{t}}^{\mathbf{H}}\right)\right)}{\underset{\boldsymbol{t} \in \mathcal{T}}{\operatorname{Mean}}\left(\left|\Lambda_{12}^{\mathbf{H}}\left(\boldsymbol{x} ; S_{\boldsymbol{t}}^{\mathbf{H}}\right)\right|\right)}, \frac{\operatorname{Var}_{\boldsymbol{t} \in \mathcal{T}}\left(\Lambda_{12}^{\mathbf{Q}}\left(\boldsymbol{x} ; S_{\boldsymbol{t}}^{\mathbf{Q}}\right)\right)}{\operatorname{Mean}_{\boldsymbol{t} \in \mathcal{T}}\left(\left|\Lambda_{12}^{\mathbf{Q}}\left(\boldsymbol{x} ; S_{\boldsymbol{t}}^{\mathbf{Q}}\right)\right|\right)},
$$

respectively. A small value of fluctuation implies a stable response, which is robust against the adverse effects introduced by the interference of closely located structures as well as image noise.

The results based on the above measurements for different combinations of noise levels and torus separations are presented and listed in Table 1 In Table 1 it is observed that both the Hessian matrix and OOF perform better when the inner-tube radii of tori rise. It is because structures having low curvature surfaces such as large innertube radius tori are easier to be detected than the tori having small inner-tube radii. To evaluate the performance drops of OOF and the Hessian matrix in handling images having closely located structures, the changes of the mean angular discrepancy and response fluctuation in various cases are investigated in Table 2 In the entries of Table 2 a small value represents high robustness against the reduction of torus separation (Table 2a); the increment of noise level (Table 2b); and both of them (Table $2 \mathrm{k}$ ).

As previously mentioned, the detection of OOF is localized at the boundary of local spherical regions. The OOF detection responses are merely induced from the intensity discontinuities taken place at the structure boundary, when the local sphere surface of OOF touches the structure boundary. In contrast to OOF, the Hessian matrix based detection relies on the computation of the weighted intensity average difference between the regions inside the structure and in the vicinity of the structure, where a nearby object is possibly present. As the correct detection scale of the Hessian matrix increases for recognizing large scale structures, the detection coverage of the correct scale of the Hessian matrix expands. It increases the chances to include adjacent structures. Hence, the increments of mean angular discrepancies and response fluctuations of the Hessian 
Table 2. The changes of mean angular discrepancy and response fluctuation from the case of $" d=5, \sigma_{\text {noise }}=0.75 "$ to other three cases presented in Table 1

(a)

\begin{tabular}{|c|c|c|c|c|}
\hline \multicolumn{5}{|c|}{ From " $d=5, \sigma_{\text {noise }}=0.75 "$ to $" d=2, \sigma_{\text {noise }}=0.75 "$} \\
\hline & \multicolumn{2}{|c|}{ Changes of mean angular discrepancy } & \multicolumn{2}{|c|}{ Changes of response fluctuation } \\
\hline $\mathrm{R}$ & Hessian matrix & $\overline{\mathrm{OOF}}$ & Hessian matrix & $\overline{\text { OOF }}$ \\
\hline 1 & +0.002 & -0.005 & +0.013 & +0.006 \\
\hline 2 & +0.073 & +0.048 & +0.052 & +0.035 \\
\hline 3 & +0.053 & +0.025 & +0.041 & +0.023 \\
\hline 4 & +0.035 & +0.024 & +0.033 & +0.031 \\
\hline 5 & +0.025 & +0.005 & +0.034 & +0.012 \\
\hline \multicolumn{5}{|c|}{ (b) } \\
\hline \multicolumn{5}{|c|}{ From " $d=5, \sigma_{\text {noise }}=0.75 "$ to $" d=5, \sigma_{\text {noise }}=1$ " } \\
\hline & \multicolumn{2}{|c|}{ Changes of mean angular discrepancy } & \multicolumn{2}{|c|}{ Changes of response fluctuation } \\
\hline $\mathrm{R}$ & Hessian matrix & $\mathbf{O O F}$ & Hessian matrix & $\mathbf{O O F}$ \\
\hline 1 & +0.112 & +0.100 & +0.050 & +0.045 \\
\hline 2 & +0.099 & +0.067 & +0.044 & +0.040 \\
\hline 3 & +0.095 & +0.059 & +0.036 & +0.010 \\
\hline 4 & +0.049 & +0.047 & +0.030 & +0.026 \\
\hline 5 & +0.053 & +0.023 & +0.021 & +0.004 \\
\hline \multicolumn{5}{|c|}{ (c) } \\
\hline \multicolumn{5}{|c|}{ From " $d=5, \sigma_{\text {noise }}=0.75 "$ to $" d=2, \sigma_{\text {noise }}=1 "$} \\
\hline & \multicolumn{2}{|c|}{ Changes of mean angular discrepancy } & \multicolumn{2}{|c|}{ Changes of response fluctuation } \\
\hline $\mathrm{R}$ & Hessian matrix & $\overline{\mathrm{OOF}}$ & Hessian matrix & $\overline{\mathrm{OOF}}$ \\
\hline 1 & +0.126 & +0.104 & +0.068 & +0.052 \\
\hline 2 & +0.203 & +0.139 & +0.106 & +0.079 \\
\hline 3 & +0.170 & +0.090 & +0.085 & +0.039 \\
\hline 4 & +0.118 & +0.062 & +0.068 & +0.054 \\
\hline 5 & +0.103 & +0.037 & +0.054 & +0.029 \\
\hline
\end{tabular}

matrix are larger than those of OOF, especially when $R$ increases, in the cases that the torus separation is reduced from 5 voxel length to 2 voxel length (the second and the forth columns versus the first and the third columns of Table 2 a).

Moreover, in the situation where noise is increased (Table 2b), it is observed that OOF (the second and the forth columns) has less increment of the mean angular discrepancies than the Hessian matrix (the first and the third columns), particularly when $R$ increases. Although the Gaussian smoothing taken by the Hessian matrix partially eliminates noise from the image volume, the smoothing process also reduces the edge sharpness of the structure boundaries. In particular, the scale factor of the Gaussian smoothing process of the Hessian matrix has to rise to deal with large scale structures. Consequently, the Hessian matrix performs detection based on the smoothed object boundaries which are easier to be corrupted by image noise. For OOF, the detection does not require Gaussian smoothing using a large scale factor ( $\sigma=1$ for OOF). It retains the edge sharpness of the structure boundaries. Therefore, the OOF detection has higher robustness against image noise than the Hessian matrix. As expected, when the torus separation is reduced to 2 voxel length and the noise level is raised to $\sigma_{\text {noise }}=1$, OOF has higher robustness than the Hessian matrix, against the presence of both closely located adjacent structures and high level noise than the Hessian matrix (Table 2k).

To summarize the results of the synthetic data experiments (Tables 1 and 2), OOF is validated in several aspects, the structure direction estimation accuracy, the stability of responses, the robustness against the disturbance introduced by closely located structures and the increment of noise levels. In some applications, an accurate structure direction estimation is vital. For instance, vascular image enhancement, the estimated 
structure direction is to avoid smoothing along the directions across object boundaries. Furthermore, for tracking curvilinear structure centerlines (a centerline tracking example is in [1]), estimated structure direction is to guide the centerline tracking process. Also, small response fluctuation facilitates the process to extract curvilinear structures or locate object centerlines by discovering the local maxima or ridges of the response.

On the other hand, the structure direction estimation accuracy and the stability of structure responses of OOF are robust against the reduction of structure separation and the increment of noise levels. As such, employing OOF to provide information of curvilinear structures is highly beneficial for curvilinear structure analysis.

\subsection{Application Example - Blood Vessel Extraction}

In this section, we demonstrate an example on utilizing OOF to supply information of curvilinear structures for extracting vessels in a vascular image. The vascular image utilized in this example is a phase contrast magnetic resonance angiographic (PCMRA) image volume (Figure 4 ) and the image intensity represents the blood flow speed inside the vasculature. The challenges to extraction algorithms are the presence of closely located vessels due to the complicated geometry of vascular structures, and the small and low intensity vessels in images with relatively high background noise level.

To perform comparison between OOF and the Hessian matrix, we replace the Hessian matrix based information used by a vessel extraction measure with the similar information extracted from OOF. It is reminded that the main goal of this paper is to propose $\mathrm{OOF}$ as a general curvilinear structure detector. Therefore, measures having heuristic parameters which involve different values for certain kinds of structures are not preferred in this example, such as the vesselness measure [6] or majority of techniques in [12] for integrating multiple eigenvalues which involve heuristic parameters. On the other hand, the sum of the first two eigenvalues employed in the synthetic experiments is designed to provide responses at centerlines of curvilinear structures. It is not suitable for vessel extraction, which requires a measure to give vessel detection responses in the entire image region. We make use of the geometric mean of the first two eigenvalues, which was proposed for the detection of vessels in [127],

$$
\mathcal{M}(\boldsymbol{x} ; s)= \begin{cases}\sqrt{\left|\lambda_{1}(\boldsymbol{x} ; s) \lambda_{2}(\boldsymbol{x} ; s)\right|}, & \lambda_{1}(\boldsymbol{x} ; s) \leq \lambda_{2}(\boldsymbol{x} ; s)<0, \\ 0, & \text { otherwise, }\end{cases}
$$

This measure is computed in a set of discrete scales to obtain the object scales, $S_{\boldsymbol{x}}^{\mathbf{H}}=$ $\arg \max _{s \in E^{\prime}}\left(\frac{s^{2}}{3} \mathcal{M}^{\mathbf{H}}\left(\boldsymbol{x} ; \frac{s}{\sqrt{3}}\right)\right)$ for Hessian matrix and $S_{\boldsymbol{x}}^{\mathbf{Q}}=\arg \max _{s \in F^{\prime}}\left(\frac{1}{4 \pi s^{2}} \mathcal{M}^{\mathbf{Q}}(\boldsymbol{x} ; s)\right)$ for OOF. There are 15 radii and scales being employed for $F^{\prime}$ and $E^{\prime}$ respectively to cover the vessel radii ranged from 1 to 8 voxel length. Linear radius samples for $F^{\prime}$ and logarithmic scale samples for $E^{\prime}$ are utilized analogous to those described in the synthetic experiments. The vessel measure response is retrieved as the resultant values of Equation 14 obtained in the estimated object scales. The binary extraction results are obtained by thresholding the vessel measure responses. The thresholding value is found empirically so that neither over-segmentation nor under-segmentation of major vessels is observed and the same amount of voxels for both the methods are selected. Finally, $4 \%$ of voxels having the highest vessel measure responses among all voxels are thresholded as the extraction results. 
The vessel extraction results are shown in Figures $4 \mathrm{~b}$ and $\mathrm{c}$. The interesting positions in the results are highlighted by the numbered arrows in Figures $4 \mathrm{~b}$ and c. In the regions pointed at by the fifth and sixth arrows in Figures $4 \mathrm{~b}$ and $\mathrm{c}$, the Hessian based method misidentifies closely located vessels as merged structures. On the contrary, the OOF based method is capable of discovering the small separation between the closely located vessels. This result is consistent with the findings in the synthetic experiments, where OOF is more robust than the Hessian matrix when handling closely located structures (Table 2a).

In Figure 4k, it is found that several vessels with weak intensity (arrows 1, 2, 3, 4 and 7) are missed by the Hessian based method where the OOF based method has no problem to extract them (Figure $4 \mathrm{~b}$ ). The reason is that the noise level relative to the weak intensity structures is higher than those relative to strong intensity structures.

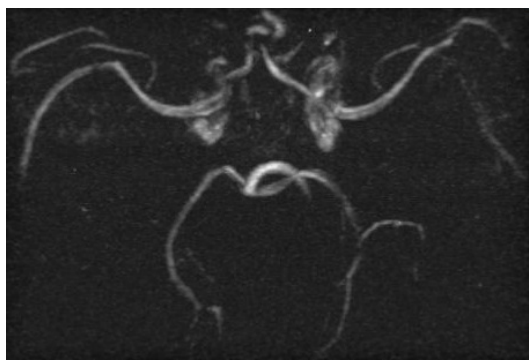

Axial view

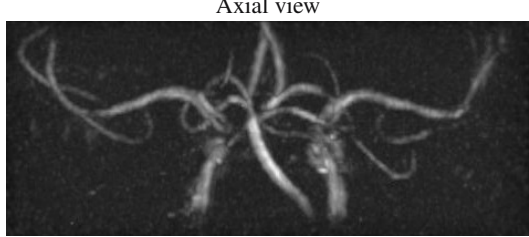

Coronal view
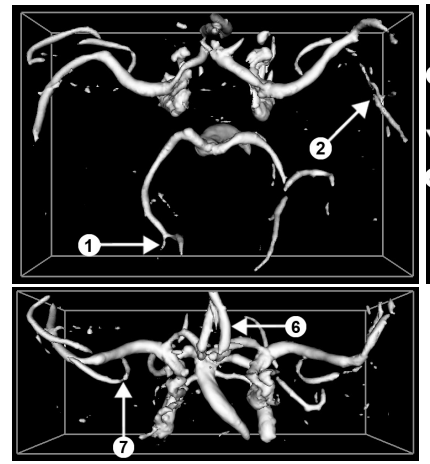

(b)

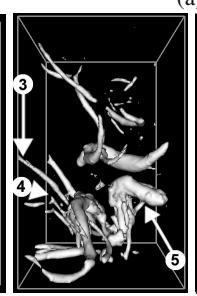

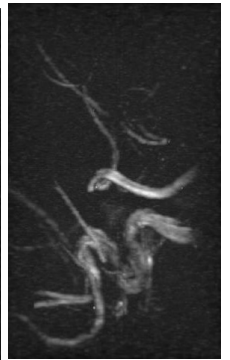

Sagittal view

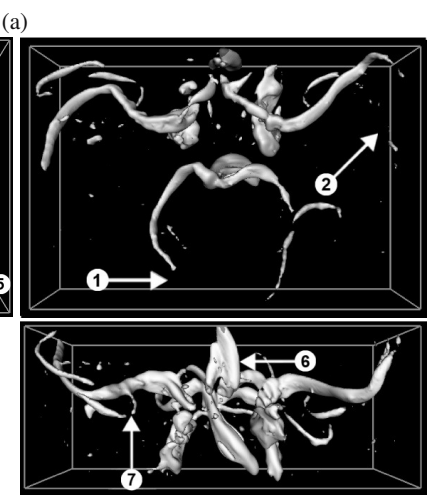

(c)

Fig. 4. (a) A phase contrast magnetic resonance angiographic image volume with the size of $213 \times 143 \times 88$ voxels. (b) The vessel extraction results obtained by using the optimally oriented flux based method. (c) The vessel extraction results obtained by using the Hessian matrix based method. 
Coherent to the synthetic experiments, in which OOF shows higher robustness against image noise as compared to Hessian matrix (see Table 2b). The vessel extraction results in this real case experiment reflects that robustness against image noise is important on extracting vessels with weak intensity.

\section{Future Developments and Conclusion}

In this paper, we have presented the use of optimally oriented flux (OOF) for detecting curvilinear structures. With the aid of the analytical Fourier expression of OOF, no discretization and orientation sampling are needed. It therefore leads to a highly efficient computation of OOF. Computation-wise, it has the same complexity as in the computation of the commonly used approach, Hessian matrix. Furthermore, computation of OOF is based on the image gradient at the boundary of local spheres. It focuses on the detection of intensity discontinuities occurred at the object boundaries of curvilinear structures.

The OOF based detection avoids including the adjacent objects. Thus, it exhibits the robustness against the interference introduced by closely located adjacent structures. This advantage is validated and demonstrated by a set of experiments on synthetic and real image volumes. In addition, in the experiments, it is observed that OOF has higher structure direction estimation accuracy and stable detection responses under the disturbance of high level image noise. With the aforementioned high detection accuracy and robustness, OOF as opposed to the Hessian matrix, to supply information of curvilinear structures, is more beneficial for curvilinear structure analysis.

In this paper, our current focus is on formulating OOF as a general detector for extracting reliable information of curvilinear structures. Identifying branches, high curvature curvilinear structures or distinguishing between blob-like, sheet-like and tubular structures would involve post-processing steps of the information extracted by the curvilinear structure detector, such as those presented in [12]. Considering the robustness of OOF against image noise and interference of closely located adjacent structures, tailoring appropriate post-processing steps of OOF for various kinds of structures will be an interesting direction for the future developments of this work.

\section{References}

1. Aylward, S., Bullitt, E.: Initialization, noise, singularities, and scale in height ridge traversal for tubular object centerline extraction. TMI 21(2), 61-75 (2002)

2. Bouix, S., Siddiqi, K., Tannenbaum, A.: Flux driven fly throughs. CVPR 1, 449-454 (2003)

3. Bouix, S., Siddiqi, K., Tannenbaum, A.: Flux driven automatic centerline extraction. MedIA 9(3), 209-221 (2005)

4. Bracewell, R.: The Fourier Transform and Its Application. McGraw-Hill, New York (1986)

5. Dimitrov, P., Damon, J.N., Siddiqi, K.: Flux invariants for shape. CVPR 1, I-835-I841(2003)

6. Frangi, A., Niessen, W., Viergever, M.: Multiscale vessel enhancement filtering. In: Wells, W.M., Colchester, A.C.F., Delp, S.L. (eds.) MICCAI 1998. LNCS, vol. 1496, pp. 130-137. Springer, Heidelberg (1998) 
7. Koller, T., Gerig, G., Szekely, G., Dettwiler, D.: Multiscale detection of curvilinear structures in 2-d and 3-d image data. In: IEEE International Conference on Computer Vision, pp. 864869 (1995)

8. Krissian, K.: Flux-based anisotropic diffusion applied to enhancement of 3-d angiogram. TMI 21(11), 1440-1442 (2002)

9. Krissian, K., Malandain, G., Ayache, N., Vaillant, R., Trousset, Y.: Model-based multiscale detection of 3d vessels. CVPR 3, 722-727 (1998)

10. Lindeberg, T.: Edge detection and ridge detection with automatic scale selection. IJCV 30(2), $117-156$ (1998)

11. Manniesing, W.N.R., Viergever, M.A.: Vessel enhancing diffusion a scale space representation of vessel structures. MedIA 10(6), 815-825 (2006)

12. Sato, Y., Nakajima, S., Shiraga, N., Atsumi1, H., Yoshida, S., Koller, T., Gerig, G., Kikinis, R.: Three-dimensional multi-scale line filter for segmentation and visualization of curvilinear structures in medical images. MedIA 2(2), 143-168 (1998)

13. Schey, H.M.: div, grad, curl, and all that, 3rd edn. W.W.Norton \& Company (1997)

14. Steger, C.: An unbiased detector of curvilinear structures. PAMI 20(2), 113-125 (1998)

15. Vasilevskiy, A., Siddiqi, K.: Flux maximizing geometric flows. PAMI 24(12), 1565-1578 (2002) 